\title{
Caries removal in primary teeth using Papacarie
}

\author{
Abstracted from \\ Deng Y, Feng G, Hu B, Kuang Y, Song J. \\ Effects of Papacarie on children with dental caries in primary teeth: a systematic review and meta-analysis. \\ Int J Paediatr Dent 2018; 28: 361-372. doi: 10.1111/ipd.12364. [Epub ahead of print] Review.PubMed PMID: 29682851. \\ Address for correspondence: Jinlin Song, Stomatological Hospital affiliated to Chongqing Medical University, \\ Chongqing 401147, China. E-mail: soongj|@163.com
}

\section{Question: Is Papacarie more effective than conventional drilling for caries removal in primary teeth?}

Data sources PubMed, Embase, Cochrane Central Register of Controlled Trials and the Web of Science were searched. Whether any limits on language were applied remains unclear. Studies published up to January 2018 were included.

Study selection Two reviewers independently selected randomised or controlled clinical trials (RCTs, CCTs) investigating carious tissue removal using Papacarie, an enzyme-based chemomechanical method, versus 'conventional' techniques in primary molars in children or adolescents.

Data extraction and synthesis Data were abstracted independently by two reviewers and risk of bias assessed. Three outcomes, bacterial counts after carious tissue removal (measure: $\log 10$ colony-forming units [CFU]), pain (measured on Wong-Baker scale) and the time needed for carious tissue removal (in seconds), were submitted to meta-analysis (effect estimate: weighted means).

Results Six RCTs and four CCTs, published 2009-2016, were included. After Papacarie-based removal, fewer bacteria remained compared with conventional treatment (MD $0.57 \log 10 \mathrm{CFU}, 95 \% \mathrm{CI}$ 0.04 to 1.09 , based on two studies). Pain was also significantly lower in the Papacarie group (-1.01, -1.72 to -0.30 , based on three studies). Papacarie-based removal took significantly longer (200.8 seconds, 152.5 to 249.1 , based on seven studies).

Conclusions Papacarie-based carious tissue removal is efficacious for bacteria removal, and exerts significantly less pain than conventional removal. However, treatment times are longer.

\section{Commentary}

This systematic review assessed an ongoing topic in cariology and paediatric dentistry, chemomechanical versus conventional (mainly bur-assisted) carious tissue removal. Chemomechanical removal, for example using hypochlorite, has been found to be more comfortable for patients and to remove carious tissue more selectively, but to require more time. ${ }^{1}$ Enzyme-based removal via Papacarie is an alternative chemomechanical strategy. The review is of relevance for practitioners. Its findings are confirmative to previous reviews; removal using Papacarie is more comfortable/less painful to patients, but takes longer. The relevance of the observed larger bacterial reduction may be questioned in light of both the understanding that sealed bacteria inactivate and the magnitude of this reduction. ${ }^{2}$

A number of methodological concerns apply, however. ${ }^{1}$ The search sequence remains unclear, as the authors do not clarify if and how terms were combined. Given that only 200 entries were yielded from all three databases, a relatively specific search seems to have been conducted. ${ }^{2}$ The restriction to primary molars (and generally, primary teeth) is not sufficiently justified. While of course, exerting less pain is highly relevant in children, it may well also be relevant in adults. ${ }^{3}$ The use of weighted means as effect measure in the meta-analysis led to the need to unnecessarily exclude studies, eg those using other pain scales than the WongBaker scale. Generally, meta-analyses are supported by only very few studies, especially towards the advantages of Papacarie. ${ }^{4}$ The authors judged the risk of bias of most studies as low; and not a single study had any item judged as high risk of bias. This is unusual and may not be fully justified; neither operators nor patients nor, for some outcomes, examiners will have been fully blinded. This lack of blinding - while admittedly not always avoidable - may have introduced considerable bias and should be reflected in risk of bias assessments accordingly. ${ }^{3,4}$ Last, the investigated outcome set was narrow, with bacterial counts being a relatively poor surrogate for clinically meaningful long-term outcomes (like pulp damage, lesion progression, restoration survival). This cannot be ascribed to this review, but rather the primary studies.

Based on this review, we may have limited confidence in the advantages of Papacarie for carious tissue removal, while there are substantive data on the additional time needed. To convince dentists to adopt this method, more and longer term data may be needed.

Falk Schwendicke

Department of Operative and Preventive Dentistry, Charité Universitätsmedizin Berlin, Germany

1. Hamama HH, Yiu CK, Burrow MF, King NM. Systematic review and metaanalysis of randomized clinical trials on chemomechanical caries removal. Oper Dent 2015; 40: E167-178

2. Oong EM, Griffin SO, Kohn WG, Gooch BF, Caufield PW. The effect of dental sealants on bacteria levels in caries lesions: a review of the evidence. I Am Dent Assoc 2008; 139: 271-278.

3. Hrobjartsson A, Emanuelsson F, Skou Thomsen AS, Hilden J, Brorson S. Bias due to lack of patient blinding in clinical trials. A systematic review of trials randomizing patients to blind and nonblind sub-studies. Int J Epidemio/ 2014; 43: 1272-1283.

4. Page MJ, Higgins JP, Clayton G, Sterne JA, Hrobjartsson A, Savovic J. Empirical evidence of study design biases in randomized trials: systematic review of meta-epidemiological studies. PloS One 2016; 11: e0159267.

Evidence-Based Dentistry (2018) 19, 74. doi:10.1038/sj.ebd.6401321 